\title{
RETINAL BLOOD VESSEL EXTRACTION AND OPTICAL DISC REMOVAL
}

\author{
R.Sai Prakash ${ }^{1}$, R.Aditya ${ }^{2}$, Y.Sameer ${ }^{3}$, S.Parameswari ${ }^{4}$, G.Santhi Kumari ${ }^{5}$ \\ ${ }^{1}$ Student, Department of ECE, Lendi Institute of Engineering and Technology, Vizianagaram, India \\ ${ }^{2}$ Student, Department of ECE, Lendi Institute of Engineering and Technology, Vizianagaram, India \\ ${ }^{3}$ Student, Department of ECE, Lendi Institute of Engineering and Technology, Vizianagaram, India \\ ${ }^{4}$ Student, Department of ECE, Lendi Institute of Engineering and Technology, Vizianagaram, India \\ ${ }^{5}$ Assistant professor, Department of ECE, Lendi Institute of Engineering and Technology, Vizianagaram, India
}

\begin{abstract}
Retinal image processing is an important process by which we can detect the blood vessels and this helps us in detecting the DIABETIC RETINOPATHY at a early stage and this is very helpful because the symptoms are not known by anyone unless we have blur eye sight or we get blind. And this mainly occurs in people suffering from high diabetes. So by extracting the blood vessels using the algorithm we can see which blood vessels are actually damaged. So by using the algorithm we can continuously survey the situation and can protect our eye-sight.
\end{abstract}

Keywords: field of view, retinopathy, thresholding, morphology, Otsu's algorithm, MATLAB. - ****

\section{INTRODUCTION}

The morphology of the retinal blood vessel and the optic disk is an important structure indicator for assessing the presence and severity of retinal diseases such as diabetic retinopathy, hypertension, glaucoma, hemorrhages, vein occlusion, and neovascularization. However to understand and to know the diameter and structure of the retinal blood vessel its time taking and also includes human errors.

A process is implemented in which we can separate the blood vessels from the optical disc and this could be done through thresholding and segmentation. The process also includes a acquisition of funds retinal images under different conditions of illumination, revolution and field of view (FOV), and the overlapping tissue in the retina cause a significant degradation of the performance of automated blood vessel and optic disk segmentations. Thus, there is a need for reliable vascular tree extraction and optic disk detection, which preserves various vessel and optic disk shapes. In the following segment, we briefly review the previous studies on the blood vessel segmentation and optic disk segmentation separately.

The appearance of the vessel is enhanced using detection processes such as morphological preprocessing techniques and adaptive filtering. The second operation is recognition of the vessel structure using thinning or branch-point operations to classify a pixel as vessel or background $(\mathrm{Bg})$. These approaches process every pixel in the image and apply multiple operations on each pixel. Some pixel processing methods use neural networks and frequency analysis to define pixels in the image as vessel pixels and $\mathrm{Bg}$ pixels.
In our optic disk segmentation process, the convergence feature of vessels into the optic disk region is used to estimate its location. We then use two automated methods [Markov random field (MRF) image reconstruction and compensation factor] to segment the optic disk.

\section{PRE-PROCESSING STAGE}

\subsection{Image Acquisition}

Contrast- enhancement method that work significantly better than regular histogram equalization for most images

\subsection{Converting Colors from RGB to HSI}

In digital image, the input image can be the RGB (Red, Green, and Blue) images or other. The RGB image can be described as M x N x 3 array of color pixels. In this paper, the RGB input image is transformed into HSI color space for further processes. $\mathrm{H}$ component of each RGB pixel is obtained using the following equation [1]

$$
\begin{gathered}
\mathrm{H}=\left\{\begin{array}{c}
\theta \text { if } B \leq G \\
360-\theta \text { if } B>G
\end{array}\right\}-(1) \\
\theta=\cos ^{-1}\left\{\left(\frac{1}{2}\right)[(R-G)+(R-B)]\right. \\
\left.\quad /\left[(R-G)^{2}+(R-G)(G-B)\right] 2\right\}
\end{gathered}
$$

The saturation component is

$$
S=\left(\frac{3}{r+g+b}\right)[\min (R, G, B\}-[2]
$$


Finally, the intensity component is

$$
I=\left(\frac{1}{3}\right)(R+G+B)-[3]
$$

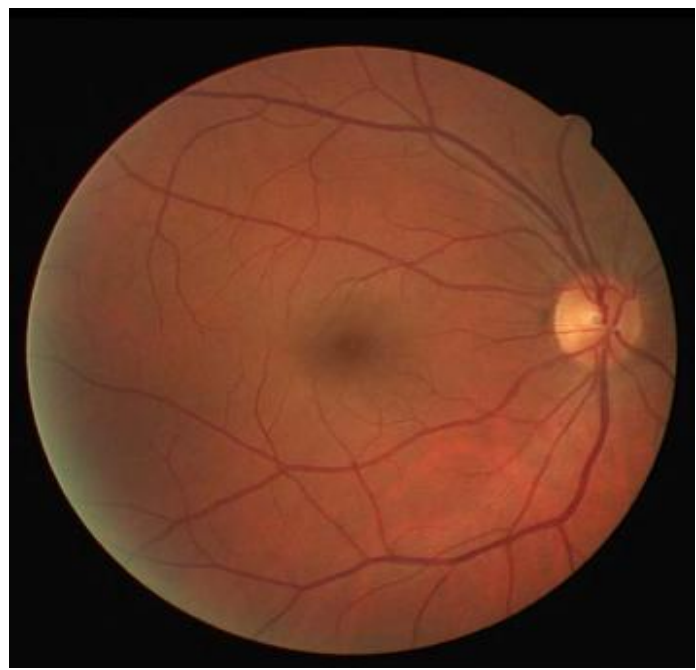

Fig: 2(a) Original image

All digital retinal images are taken from patients using the non-mydriatic retinal fundus [6] camera. The images are stored in JPEG image format file (.jpg) and taken from "Eye and ENT General Hospital (Mandalay)". In this research, the retinal images are taken from the "Eye and ENT General Hospital (Mandalay)" and also from the websites.

The original (RGB) image is transformed into appropriate color space for further processes. And then, filtering technique is used to reduce the effect of noise. After using the filtering technique, the noise such as salt and pepper noise are removed from the image. Then contrast-limited adaptive histogram equalization (CLAHE) is used for image enhancement. Unlike histogram, it operates on small data regions rather than the entire image. This function uses a

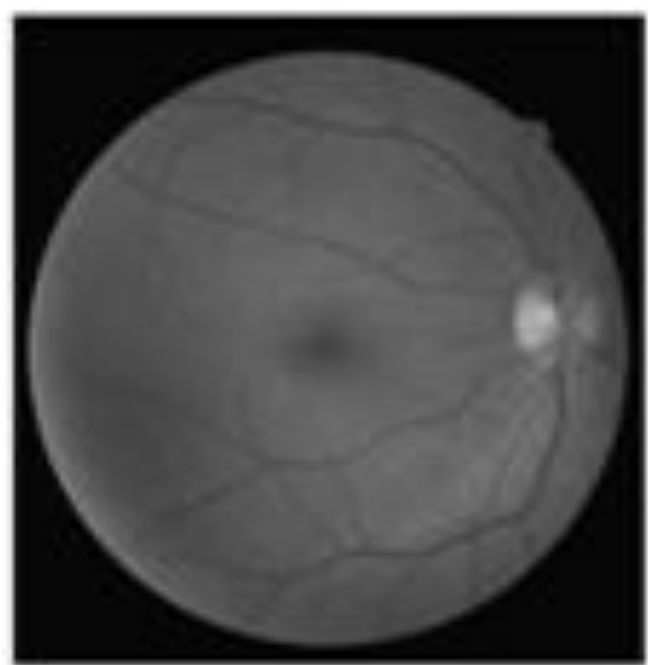

Fig 2(b): HSI image

Fig-2(b): shows the result of the converting color from RGB to HIS image.

\section{OPTICAL DISC REMOVAL}

\subsection{Thresholding}

The Otsu's thresholding technique is applied to the image to detect the desire area.

Equations of Otsu algorithm are

$$
\begin{array}{cc}
\sigma_{\text {Between }}^{2}(\mathrm{~T})=\mathrm{w}_{\mathrm{B}}(\mathrm{T}) \mathrm{w}_{\mathrm{o}}(\mathrm{T})\left[\mu_{\mathrm{B}}(\mathrm{T})-\mu_{\mathrm{o}}(\mathrm{T})\right]^{2} \\
\mathrm{w}_{\mathrm{B}}(\mathrm{T})=\sum_{i=T}^{L-1} p(i) \quad, \mu_{\mathrm{B}}=\sum_{i=T}^{L-1}\left(\frac{i p(i)}{p(i)}\right) \\
\mathrm{w}_{0}(\mathrm{~T})=\sum_{i=T}^{L-1} p(i) \quad, \mu_{\mathrm{O}=} \sum_{i=T}^{L-1}\left(\frac{i p(i)}{p(i)}\right)_{-[4]}
\end{array}
$$

$\sigma_{\text {Between }}^{2}(\mathrm{~T})=$ Between-class variance

$\mathrm{w}=$ weight, $\mathrm{B}=$ background of the image, $\mathrm{o}=$ object of image $\mu=$ combined mean,

$\mathrm{T}=$ threshold value

The optic disc is the largest and brightest region of the image. The optic disc detection is useful because it can reduce the false positive detection of the exudates. Fig.5 shows the general flow chart of the optic disc detection.

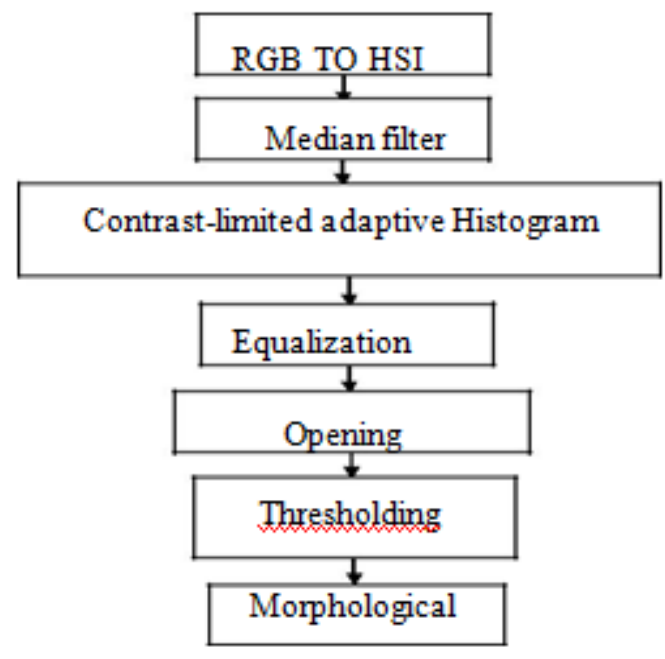

Fig:3(a) Flow Chart of Optic disc Detection

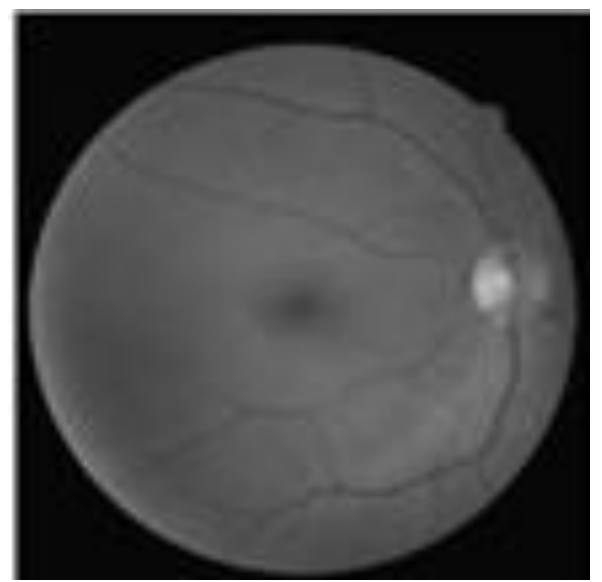

Fig:3(b) opened image 


\section{BLOOD VESSELS DETECTION}

The blood vessels detection and elimination is also important as the optic disc detection for further process because the optic disc and the blood vessels are the normal features of the image.

\subsection{Smoothening with Level Sets}

Initially, the images are filtered using two-dimensional matched filters. The filter employs three primary principles. First, pairs of blood vessel walls may be approximated as linear due to typically small curvature. Second, vessels appear darker than the background as a result of low reactance in comparison to other retinal surfaces. The problem may be modeled as a Gaussian. Finally, the width of blood vessels is variable. Precisely, the algorithm is as follows. A kernel is generated in the following fashion: $P=$ $\left[\begin{array}{ll}x & y\end{array}\right]$ is a point in the kernel and $\Theta i$ is the orientation. The kernel is centered on the origin, $P=[00]$.[4]

$$
\mathrm{ri}=\left[\begin{array}{cc}
\cos \theta i & \sin \theta i \\
\sin \theta i & \cos \theta i
\end{array}\right]-[5]
$$

The rotation matrix [5], is used, and $p i=$ Pri. Twelve different kernels are generated for an angular resolution of 15 degrees. Each kernel is convolved with the image, and at each point within the image, the maximum response is considered.

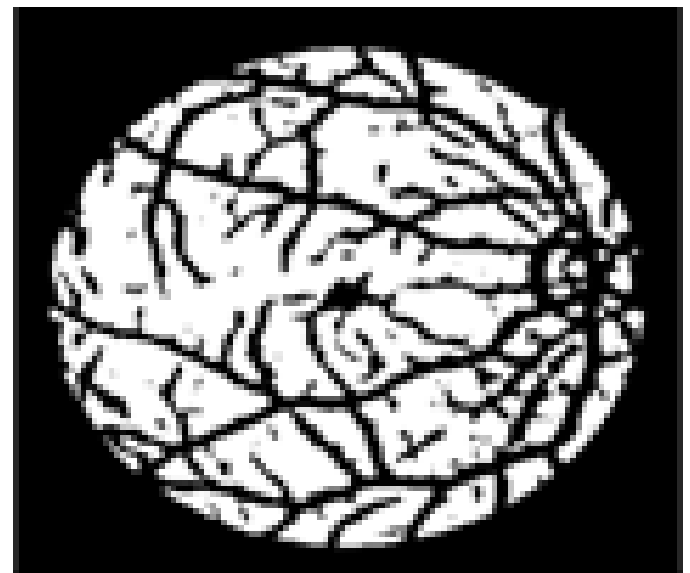

Fig:4(a) blood vessel extracted using Gaussian kernel normalization

\subsection{Hybrid Retinal Image Registration}

[6]First, co-occurrence matrix definition to increase the local entropy. The co-occurrence matrix of an image show the intensity transitions between adjacent pixels. The original co- occurrence matrix is asymmetric by considering the horizontally right and vertically lower transitions. We want to add some jittering effect to the co-occurrence matrix that tends to keep the similar spatial structure but with much less variations, i.e., $\mathrm{T}=[\mathbf{t} \mathbf{i}, \mathbf{j}] \mathbf{N} \times \mathbf{N}$ is computed as follows and also shown in Fig. 4(a).

- For every pixel $(1, \mathrm{k})$ in an image $\mathbf{I}$

- $\mathbf{i}=\mathbf{I}(1, \mathrm{k})$;
- $\mathbf{j}=\mathbf{I}(\mathrm{l}, \mathrm{k}+1)$

$-\mathrm{d}=\mathrm{I}(\mathrm{l}+1, \mathrm{k}+1)$

- $\mathbf{t i j}=\mathbf{t i d}+1$

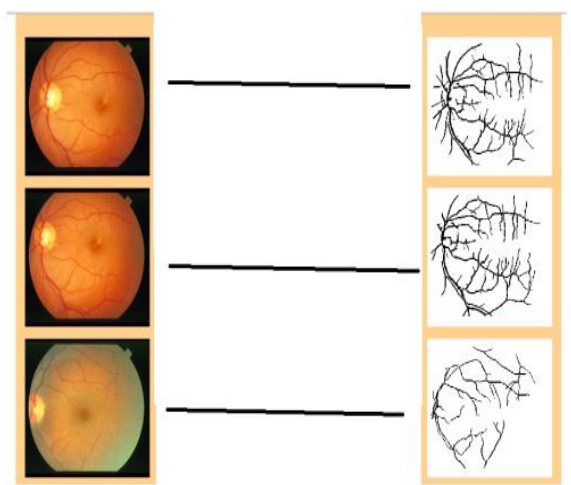

Fig:4(b) few samples of the output

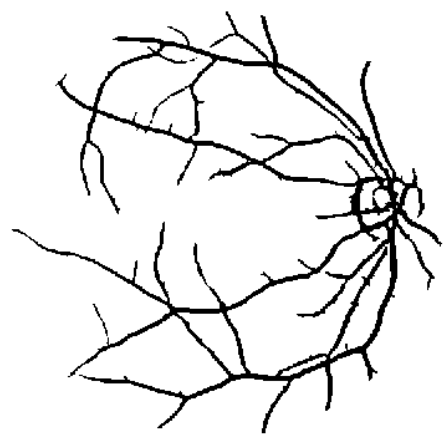

Fig:4(c) Output of blood vessel extraction.

\section{DISCUSSION}

In this paper, the mathematical morphology is applied to detect the optic disc and the blood vessels. Detection of optic disc and the blood vessels is the important step for further processes in the detection of diabetic retinopathy. And also it helps the ophthalmologists to detect the optic disc and the vessels more easily and faster. Even on a poor computer system, this technique can work effectively. This method can work faster and it can process within a few minutes. Therefore, this method is suitable for rural area in developing countries.

\section{CONCLUSION}

Mathematical morphology method is used for optic disc and the blood vessels detections. For these detections, the input images are taken from the websites and "Eye and ENT General Hospital (Mandalay). The input image is in RGB color space and for the further processes the image is converted into appropriate color space. The median filter, averaging filter and the wiener filter are used for the noise reduction. Among these filter, median filter is chosen for the 
filtering purpose because median filter can reduce the effect of noise without blurring the edge. And then, the adaptive histogram equalisation technique is used for image enhancement. It is also used to overcome the uneven illumination case. Therefore, median filter and the adaptive histogram equalisation techniques are used for noise reduction and image enhancement purposes. Optic disc detection and the blood vessels detection are the major role in the screening of eye diseases. The results of this work can be used in the future.

\section{REFERENCES}

[1]. A.M. Mendonca, A. Campilho, "Segmentation of retinal blood vessels by combining the detection of centerlines and morphological reconstruction," IEEE Trans. Med. Imag., vol. 25, pp. 1200-1213, 2006.

[2]. B. Zhang, L. Zhang, L. Zhang, F. Karray, " Retinal vessel extraction by matched filter with first-order derivative of Gaussian," Computers in Biology and Medicine, vol. 40, pp. 438-445, 2010.

[3]. E. Ricci and R. Perfetti, "Retinal blood vessel segmentation using line operators and support vector classification," IEEE Trans. Med. Imag., vol. 26, no. 10, pp. 1357-1365, Oct. 2007.

[4]. M.M. Fraza, P. Remagninoa, A. Hoppea, B. Uyyanonvarab, A.R. Rudnickac, C.G. Owenc, S.A. Barmana, "Blood vessel segmentation methodologies in retinal images A survey", Computer Methods and Programs in Biomedicine Volume 108, Issue 1, October 2012, Pages 407-433

[5]. A.D. Hoover, V. Kouznetsova, M. Goldbaum, Locating blood vessels in retinal images by piecewise threshold probing of a matched filter response, IEEE Transactions on Medical Imaging 19 (2000) 203-210.

[6]. F. Oloumi, R. M. Rangayyan, F. Oloumi, P. Eshghzadeh-Zanjani, and F. J. Ayres, "Detection of Blood Vessels in Fundus Images of the Retina using Gabor Wavelets," In Proc. IEEE Engineering in Medicine and Biology Society(EMBS) 29th Annual International Conference, August ,2007,pp. 6451-6454. 